\title{
Comparison of Vonoprazan and Amoxicillin Dual Therapy with Standard Triple Therapy with Proton Pump Inhibitor for Helicobacter Pylori eradication: A Randomized Control Trial
}

\author{
Bader Faiyaz Zuberi ${ }^{1}$, Faiza Sadaqat Ali ${ }^{2}$, Tazeen Rasheed ${ }^{3}$, \\ Nimrah Bader ${ }^{4}$, Sana Muhammad Hussain ${ }^{5}$, Anoshia Saleem ${ }^{6}$
}

\begin{abstract}
Objectives: To compare the efficacy of Vonoprazan based dual treatment versus PPI based treatment for the eradication of Helicobacter pylori infection.

Methods: A randomized controlled trial was conducted in Department of Medicine/Gastroenterology Ruth KM Pfau Civil Hospital, DMC during the period of 22 June to 21 September 2021. Sample size was calculated as 96 in each Group. All patients of age 18-75 years with Helicobacter Pylori Infection were inducted and randomly allocated to two groups. Group-A: were given Capsule Amoxicillin $1 \mathrm{gm}$; Tablet Clarithromycin $500 \mathrm{mg}$; Capsule Omeprazole $20 \mathrm{mg}$ all medications were given twice daily for two weeks. Group-B were given Capsule Amoxicillin $1 \mathrm{gm}$; Tablet Vonoprazan 20 mg (Vonozon ${ }^{\odot}$, m/s Getz Pharma, Pakistan) twice daily for two weeks. Confirmation of Hp eradication was done by stool Hp antigen test four weeks after completion of treatment. Nine and four patients were lost to follow-up in Group-A \& B respectively. Analysis was conducted on 87 patients in Group-A and 92 patients in Group-B.

Results: Out of eighty-seven patients in Group-A and ninety-two patients in Group-B, 73 (83.9\%) patients in Group-A and $86(93.5 \%)$ patients in Group-B had negative $\mathrm{H}$ pylori result respectively after treatment $(p=$ .042 ). Significantly higher frequencies of adverse events were observed in Group-A as compared to Group-B in nausea/vomiting $(p=.035)$ and bloating $(p=.045)$.
\end{abstract}

Conclusion: VA-dual provides an acceptable eradication rate with fewer adverse events.

KEYWORDS: Helicobacter Pylori; Vonoprazan; Proton Pump Inhibitors; Clarithromycin; Amoxicillin.

doi: https://doi.org/10.12669/pjms.38.4.5436

How to cite this:

Zuberi BF, Ali FS, Rasheed T, Bader N, Hussain SM, Saleem A. Comparison of Vonoprazan and Amoxicillin Dual Therapy with Standard Triple Therapy with Proton Pump Inhibitor for Helicobacter Pylori eradication: A Randomized Control Trial . Pak J Med Sci. 2022;38(4):965-969. doi: https://doi.org/10.12669/pjms.38.4.5436

This is an Open Access article distributed under the terms of the Creative Commons Attribution License (http://creativecommons.org/licenses/by/3.0), which permits unrestricted use, distribution, and reproduction in any medium, provided the original work is properly cited.

1. Bader Faiyaz Zuberi FCPS, Meritorious Professor,

2. Faiza Sadaqat Ali FCPS, Senior Registrar,

3. Tazeen Rasheed FCPS, Associate Professor,

4. Nimrah Bader MD, PGY-4 Geriatric Medicine Fellow,

University of Miami/Jackson Health System, Miami, FL, USA.

5. Sana Muhammad Hussain PG Trainee,

6. Anoshia Saleem FCSP (Gastro), Consultant,

Dr Ruth KM Pfau Civil Hospital Karachi, Pakistan.

1-3,5: Department of Medicine/Gastroenterology,

Dow Medical College, Dow University of Health Sciences,

Karachi, Pakistan.

Correspondence:

Dr. Bader Faiyaz Zuberi

C-404, Al-Habib Pride, CL-8/5,

Civil Lines, Karachi, Pakistan.

E-mail: bader@zuberi.net

* Received for Publication:

* Accepted for Publication:
October 3, 2021

February 5, 2022

\section{INTRODUCTION}

Helicobacter pylori $(\mathrm{Hp})$ is a bacteria that causes inflammation of gastric mucosa and it is a key cause of peptic ulcer globally. ${ }^{1}$ It colonizes the gastric mucosa and is guilty for a number of gastric related diseases such as chronic gastritis, gastric adenocarcinoma, and gastric mucosa associated lymphoid tissue (MALT) lymphoma. ${ }^{2}$ Prevalence of $\mathrm{Hp}$ varies from $85-95 \%$ in developing countries and from $30-50 \%$ in developed countries. ${ }^{3}$ It is advised that all patients with evidence of active infection with $\mathrm{Hp}$ should be offered eradication therapy. Multiple antibiotic regimens have been evaluated for Hp therapy. However, there are only 
few regimens which have consistently achieved high eradication "Initially triple therapy including a proton pump inhibitor (PPI) and two antibiotics such as amoxicillin with clarithromycin or metronidazole was considered as the best therapy for the Hp eradication". As a result of decreased efficacy of this treatment, other different therapies have been developed.

Major reason for decreased effectiveness was development of resistance to antibiotics and failure to maintain sustained increase in gastric $\mathrm{pH}$ of $>5.7$ during course of the day. ${ }^{4-6}$ To overcome this double dose of Proton Pump Inhibitor (PPI) are used in all $\mathrm{Hp}$ eradication regimens to maintain high $\mathrm{pH}$. Since PPI based therapy was found to be superior to non PPI based therapy for Hp eradication therefore the addition of anti-secretory drugs to antibiotic is of utmost importance in the eradication regimens. ${ }^{1}$ Vonoprazan (VPZ) is a novel potassium competitive acid blocker (PCAB). It works by competing for potassium on the luminal side of the parietal cell and causes rapid and reversible inhibition of H-K ATPase and therefore inhibits extended acid secretion. In contrast to PPIs Vonoprazan is a more potent inhibitor of acid secretion. It has a rapid onset of action, less anti-secretory variability, greater safety and better tolerability. ${ }^{1}$

Important factors in failure of $\mathrm{Hp}$ therapy is local resistance to antibiotics used and if they are taken within two weeks of start of $\mathrm{Hp}$ eradication regimen. Clarithromycin resistance is reported at $43.9 \%^{7}$ and is a major cause of treatment failure. That's why we excluded it from our current study and planned to compare standard PPI based triple therapy with Vonoprazan based dual therapy without Clarithromycin. No local studies have compared the efficacy in the eradication rates of Vonoprazan based dual therapy versus PPI based triple therapy for the eradication of Hp infection in our region. Therefore, this study was conducted to compare the efficacy of Vonoprazan based dual therapy with one antibiotic versus PPI based triple therapy with two antibiotics for the eradication of $\mathrm{Hp}$ in a randomized control setting.

Our objective was to compare the efficacy of Vonoprazan based dual treatment versus PPI based treatment for the eradication of Helicobacter pylori infection.

Operational Definition: Helicobacter Pylori infection: Hp infection will be labelled by any one of following investigation:

- Helicobacter Pylori Stool Antigen

- Histopathology on Giemsa Stain

\section{METHODS}

This Randomized control trial. was conducted in the Medical OPD and Medical Unit-1 of Dr. Ruth K.M. Pfau, Civil Hospital Karachi affiliated with Dow University of Health Sciences from 22 June 2021 till 21 September 2021

Group sample sizes of 96 in Group-A and 96 in Group-B achieve $95.246 \%$ power to detect a difference between the group proportions of 0.3 . The proportion in Group-A is assumed to be 0.5 under the null hypothesis and 0.8 under the alternative hypothesis. The proportion in Group-B is 0.5 . The test statistic used is the two-sided Z-Test with unpolled variance. The significance level of the test is $0.05 "$. Calculation was done using PASS 2019 Power Analysis and Sample Size Software (2019, NCSS, LLC. Kaysville, Utah, USA, ncss.com/ software/pass). The Sampling Technique: was Non-Probability Consecutive

Inclusion Criteria: All patients between age of 1875 years with $\mathrm{Hp}$ infection as described above.

Exclusion Criteria:

- History of gastric surgery such as partial gastrectomy.

- History of allergy to drugs used in the study.

- Intake of antibiotics, PPIs, corticosteroids within the last two weeks.

- Pregnant or lactating females.

- Alcohol or drug addiction.

- Severe neurological or psychiatric disorder.

Data collection procedure: All patients presenting to the OPD or Medical Unit -I of Dr. Ruth KM Pfau, Civil Hospital Karachi and fulfilling the inclusion criteria were included after taking informed consent.

The participants were randomly allocated to two groups with help of random number table and were treated for two weeks.

Group-A: were given:

- Capsule Amoxicillin 1 gm twice daily

- Tablet Clarithromycin $500 \mathrm{mg}$ twice a day

- Capsule Omeprazole $20 \mathrm{mg}$ twice a day

Group-B were given:

- Capsule Amoxicillin 1 gm twice daily

- Tablet Vonoprazan $20 \mathrm{mg}$ twice a day (Vonozon ${ }^{\odot}$, m/s Getz Pharma, Pakistan).

- Confirmation of Hp eradication was done by stool $\mathrm{Hp}$ antigen test 4 weeks after completion of treatment.

Data Analysis: Data was stratified according to age \& gender. Frequency of gender \& mean $( \pm S D)$ of age was compared between groups using $X^{2}$ test and Student's t-test respectively. Frequency of $\mathrm{Hp}$ eradication between two groups was compared 
using Pearson's $\chi^{2}$ test. A $p$ value $\leq .05$ was taken as significant.

\section{RESULTS}

Two hundred thirty-three patients were assessed for eligibility out of these a total of 41 patients were excluded. The breakup of these was, not fulfilling selection criteria $(n=22)$, declined to give consent $(n=16)$ and history of using PPI in preceding two weeks $(n=3)$. Randomization was continued till sample size of 96 in each group was achieved. Nine patients were lost to follow-up in Group-A, while four patients were lost to follow-up in Group-B. Analysis was conducted on 87 patients in Group-A and 92 patients in Group-B. Details of enrollment \& randomization are given in Fig.1.

Total one hundred seventy-nine patients were analyzed which included 109 males and 70 females. In Group-A there were $54(62.1 \%)$ males and 33 $(37.9 \%)$ females and in Group-B there were 55

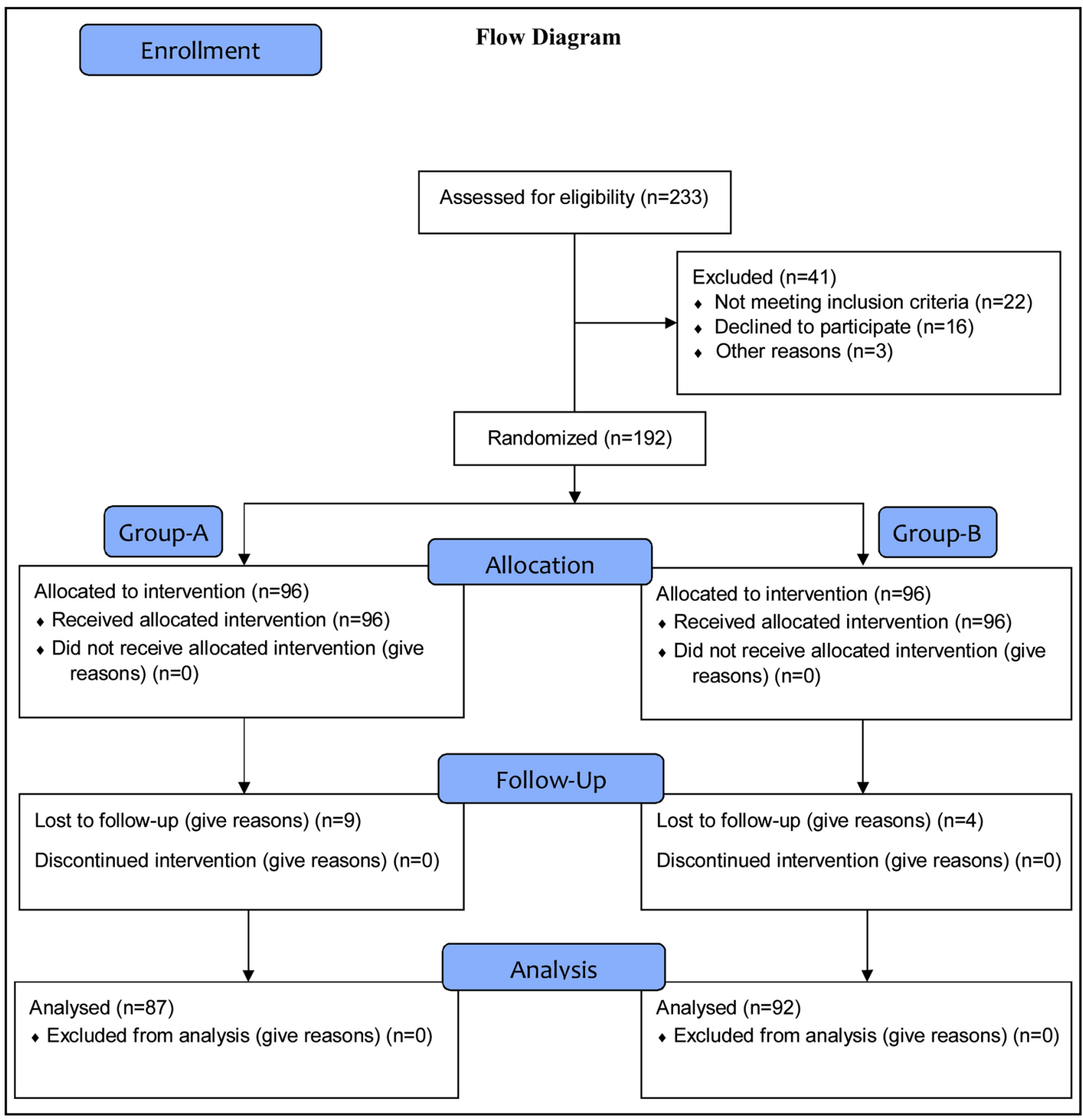

Fig.1: Randomization and Group-Allocation of patients. 
Bader Faiyaz Zuberi et al.

Table-I: Helicobacter Pylori Eradication between Groups.

\begin{tabular}{llccccc}
\hline & & \multicolumn{2}{c}{ Group } & & Statistics \\
\cline { 3 - 6 } & & Group $-A$ & Group $-B$ & $x^{2}$ & $d f$ & $p$ value \\
\hline \multirow{2}{*}{ HP Stool Ag } & Negative & $73(83.9 \%)$ & $86(93.5 \%)$ & & & \\
& Positive & $14(16.1 \%)$ & $06(6.5 \%)$ & & & \\
\hline Total & & $87(100 \%)$ & $92(100 \%)$ & & \\
\hline
\end{tabular}

$(59.8 \%)$ males and 37 (40.2\%) females. Both groups were comparable in frequency of gender $c^{2}(\mathrm{df}=1$, $\mathrm{n}=179)=.09 ; p=.754$. Mean age $\pm \mathrm{SD}$ in Group-A was $40.2 \pm 9.8$ years and in Group-B was 41.4 \pm 10.6 years respectively, statistically there was no significant difference in age between two groups $t$ $(177)=-0.81, p=.422$.

Out of eighty-seven patients in Group-A and ninety-two patients in Group-B, $73(83.9 \%)$ patients in Group-A and 86 (93.5\%) patients in Group-B had negative $H$ pylori result respectively after treatment, $\mathrm{c}^{2}(\mathrm{df}=1, \mathrm{n}=179)=4.126 ; p=.042$. Vonoprazan based dual therapy more likely to eradicate $\mathrm{H}$. pylori than convention PPI based triple therapy.

Few adverse effects were reported, but none so severe to stop treatment. Significantly higher frequencies of adverse events were observed in Group-A as compared to Group-B in nausea/ vomiting and bloating, details are given in Table-II.

\section{DISCUSSION}

To the best of our knowledge, this is the first randomized control study to show the efficacy and safety of 14 days Vonoprazan amoxicillin dual therapy. Results of our study demonstrated higher H. Pylori eradication rate of $93.5 \%$ by Vonoprazan based dual therapy as compared to PPI based conventional triple therapy, which achieved $83.9 \%$ eradication rate $(p<0.05)$. In majority of trials a 7 -day dual or triple therapy of vonoprazan was given along with either amoxicillin alone or amoxicillin plus clarithromycin to reveal the eradication rate upto $85 \%$ to $87 \% .{ }^{8}$ No trial extended upto 14 days to document further improvement in efficacy of vonoprazan.

Another important finding in our study was fewer adverse effects and easy tolerability in vonoprazan based dual therapy compared to conventional triple therapy. Only few patients in VPZ group developed nausea/ vomiting, bloating and diarrhea, but none was severe enough to stop regimen. Similarly Qiu-Ju Lyu et al., in their meta-analysis on efficacy and safety of VPZ-based versus proton pump inhibitor-based triple therapy for helicobacter pylori eradication, reported significantly less incidence of adverse effects and well tolerability in VPZ-based triple therapy than that in PPI-based triple therapy (pooled incidence, 32.7\% vs $40.5 \%$; OR, 0.71 ; 95\% CI: [0.53-0.95]; $P<0.05) .{ }^{9}$

Although Vonoprazan does not have anti-microbial properties, this significant superiority of Vonoprazan over PPI for H-Pylori eradication is suggested mainly from more sustained maintenance of gastric $\mathrm{PH}$ as VPZ $40 \mathrm{mg}$ daily can maintain $\mathrm{pH}>$ 4.0 for $>90 \%$ of the day. ${ }^{10}$ The gastric $\mathrm{H}^{+}, \mathrm{K}^{+}$-ATPase is the preferred target for acid suppression. Until recently, the only drug that effectively inhibited this ATPase was the proton pump inhibitor (PPI). PPI is a pro drug, that require acid for its activation. Once acid-activated, PPI bind to ATPase (proton pump) to inhibit acid secretion. But because of short halflife of PPI and continued de novo production of the $\mathrm{H}^{+}, \mathrm{K}^{+}$-ATPase, night-time acid secretion is not fully

Table-II: Comparison of Adverse Events between Groups with $\chi^{2}$ test.

\begin{tabular}{lccccc}
\hline & \multicolumn{2}{c}{ Groups } & \multicolumn{3}{c}{ Statistics } \\
\cline { 2 - 6 } & Group-A & Group-B & $x^{2}$ & $d f$ & p value \\
\hline Diarrhea & $9(10.3 \%)$ & $3(3.3 \%)$ & 3.588 & 1 & .058 \\
Nausea/Vomiting & $13(14.9 \%)$ & $5(5.4 \%)$ & 4.469 & 1 & .035 \\
Bloating & $11(12.6 \%)$ & $4(4.3 \%)$ & 4.008 & 1 & .045 \\
\hline
\end{tabular}


inhibited. Whereas vonoprazan, which is a potassium-competitive acid blocker (PCAB) is an active drug that does not require acid for its activation, has seven hours half-life, bind reversibly with both active $\mathrm{H}^{+}, \mathrm{K}^{+}$-ATPase pumps and Inactive pumps with slow dissociation rate. PPI require meal for its activation while Vonoprazan has no meal dependency. Onset of action of Vonoprazan is within 24 hours, $\mathrm{pH}>4$ sustained over most of the time, including overnight in majority of patients, without tolerance to the drug. ${ }^{10,11}$ This rapid, sustained acid control allows antibiotic to work efficiently at optimal PH against H-Pylori organisms.

Antimicrobial stewardship and recognizing optimal treatment and duration of therapy, without unjustified use of antibiotics is imperative in current era of antimicrobial resistance. DY Graham et al. in his study brought up the fact that vonoprazan and amoxicillin dual therapy achieved a cure rate of $80 \%$, when given in clarithromycin resistant patients. ${ }^{12}$ He further highlighted that adding clarithromycin to vonoprazan and amoxicillin as a triple therapy in general population approximately added only $12 \%$ benefit in cure rate, and $88 \%$ of the patients received clarithromycin unnecessarily. ${ }^{12}$ In his study they gave seven days dual treatment with vonoprazan and amoxicillin to achieve a cure rate of $80 \%$, while in our study we gave dual therapy for extended period of 14 days to achieved $93.5 \%$ cure rate.

One of the main strengths of our study is its study design of being randomized control that provides higher level of statistical reliability. A meta-analysis, where 16 studies were selected for quantitative review, had limitation that 15 of 16 studies in their meta-analysis were retrospective and used historical controls with a time-frame shift between the two groups,$^{13}$ whereas our study is prospective study. Almost all the studies done on vonoprazan gave seven days of treatment, in our knowledge, but we gave it for extended 14 days to fully evaluate its efficacy. Although significantly less adverse effects observed in VPZ dual therapy group, but long-term adverse effects need to be explored from more prolong and sustained acid suppression. Future studies, comparing VPZ dual therapy to VPZ triple, concomitant or sequential therapies may be needed to further evaluate improvement in H-Pylori eradication rate and to develop optimal first line eradication regimen with vonoprazan.

\section{CONCLUSION}

In the era of growing antimicrobial resistance, VA-dual therapy is a potential new first- line $\mathrm{Hp}$ therapy for cases because it provides an acceptable eradication rate and high safety and will have a potentially less negative impact on future antimicrobial resistance.

Conflict of Interest: All authors state no disclosures for any financial conflict of interest.

\section{REFERENCES}

1. Abadi ATB, Ierardi E. Vonoprazan and Helicobacter Pylori Treatment: A Lesson from Japan or a Limited Geographic Phenomenon? Front Pharmacol. 2019;10(316. doi: 10.3389/fphar.2019.00316

2. Hooi JKY, Lai WY, Ng WK, Suen MMY, Underwood FE, Tanyingoh D, et al. Global Prevalence of Helicobacter Pylori Infection: Systematic Review and Meta-Analysis. Gastroenterology. 2017;153(2):420-429. doi: 10.1053/j.gastro.2017.04.022

3. Burucoa C, Axon A. Epidemiology of Helicobacter Pylori Infection. Helicobacter. 2017;22( Suppl-1). doi: 10.1111/hel.12403

4. Munoz N, Sanchez-Delgado J, Baylina M, Lopez-Gongora S, Calvet X. Prevalence of Helicobacter Pylori Resistance after Failure of First-Line Therapy. A Systematic Review. Gastroenterol Hepatol. 2018;41(10):654662. doi: 10.1016/j.gastrohep.2018.06.014

5. Ke H, Li J, Lu B, Yang C, Wang J, Wang Z, et al. The Appropriate Cutoff Gastric Ph Value for Helicobacter Pylori Eradication with Bismuth-Based Quadruple Therapy. Helicobacter. 2021;26(1):e12768. doi: 10.1111/hel.12768

6. Wang YC, Chen YP, Ho CY, Liu TW, Chu CH, Wang HY, et al. The Impact of Gastric Juice $\mathrm{Ph}$ on the Intraluminal Therapy for Helicobacter Pylori Infection. J Clin Med. 2020;9(6). doi: 10.3390/jcm9061852

7. Alavifard H, Mirzaei N, Yadegar A, Baghaei K, Smith SM, Sadeghi A et al. Investigation of Clarithromycin Resistance-Associated Mutations and Virulence Genotypes of Helicobacter Pylori Isolated from Iranian Population: A Cross-Sectional Study. Curr Microbiol 2021;78(1):244-254. doi: $10.1007 /$ s00284-020-02295-7

8. Suzuki S, Gotoda T, Kusano C, Ikehara H, Ichijima R, Ohyauchi M, et al. Seven-Day Vonoprazan and Low-Dose Amoxicillin Dual Therapy as First-Line Helicobacter Pylori Treatment: A Multicentre Randomised Trial in Japan. Gut. 2020;69(6):1019-1026. doi: 10.1136/gutjnl-2019-319954

9. Lyu QJ, Pu QH, Zhong XF, Zhang J. Efficacy and Safety of VonoprazanBased Versus Proton Pump Inhibitor-Based Triple Therapy for Helicobacter Pylori Eradication: A Meta-Analysis of Randomized Clinical Trials. BioMed Res Int. 2019;2019.

10. Sakurai Y, Nishimura A, Kennedy G, Hibberd M, Jenkins R, Okamoto H, et al. Safety, Tolerability, Pharmacokinetics, and Pharmacodynamics of Single Rising Tak-438 (Vonoprazan) Doses in Healthy Male Japanese/ Non-Japanese Subjects. Clin Transl Gastroenterol. 2015;6(6):e94. doi: $10.1038 / \operatorname{ctg} .2015 .18$

11. Jenkins H, Sakurai Y, Nishimura A, Okamoto H, Hibberd M, Jenkins R, et al. Randomised Clinical Trial: Safety, Tolerability, Pharmacokinetics and Pharmacodynamics of Repeated Doses of Tak-438 (Vonoprazan), a Novel Potassium-Competitive Acid Blocker, in Healthy Male Subjects. Aliment Pharmacol Ther. 2015;41(7):636-648. doi: 10.1111/apt.13121. PMID 25707624

12. Graham DY, Lu H, Shiotani A. Vonoprazan-Containing Helicobacter Pylori Triple Therapies Contribution to Global Antimicrobial Resistance. J Gastroenterol Hepatol. 2021;36(5):1159-1163. doi: 10.1111/jgh.15252

13. Shinozaki S, Kobayashi Y, Osawa H, Sakamoto H, Hayashi Y, Lefor AK, et al. Effectiveness and Safety of Vonoprazan Versus Proton Pump Inhibitors for Second-Line Helicobacter Pylori Eradication Therapy: Systematic Review and Meta-Analysis. Digestion 2021;102(3):319-325. doi: $10.1159 / 000504939$

Authors' Contribution: BFZ, FSA, NB: Substantial contributions to conception and design, or acquisition of data, or analysis and interpretation of data. TR, SMH, AS: Drafting the article or revising it critically for important intellectual content BFZ: Final approval of the version to be published. NB: Statistical Analysis. Agreement to be accountable for all aspects of the work in ensuring that questions related to the accuracy or integrity of any part of the work are appropriately investigated and resolved. (All Authors). 